\title{
Author Correction: Precision genome engineering through adenine base editing in plants
}

Beum-Chang Kang, Jae-Young Yun, Sang-Tae Kim, YouJin Shin, Jahee Ryu, Minkyung Choi, Je Wook Woo and Jin-Soo Kim (1)

Correction to: Nature Plants https://doi.org/10.1038/s41477-018-0178-x, published online 4 June 2018.

In Supplementary Fig. 1b originally published with this Brief Communication, the DNA sequence of nickase Cas9 was incorrect; this has now been amended.

Published online: 23 August 2018

https://doi.org/10.1038/s41477-018-0251-5 